\title{
Interações medicamentosas potenciais em diabéticos tipo 2 participantes de um programa de educação em saúde
}

\author{
Potential drug interactions in participants of a health education program, presenting \\ type 2 diabetic
}

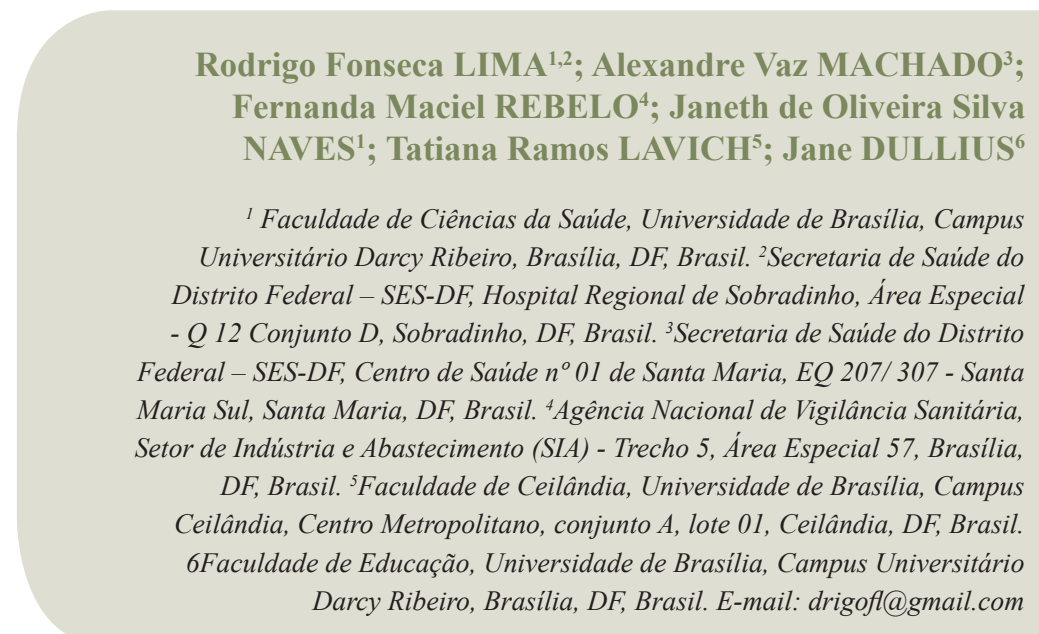

ABSTRACT

Cross-sectional study in order to determine the prevalence, clinical impact and factors associated with Potential Drug Interactions (PDI) in participants with type 2 diabetes (DM2) of a health education program. This study involved 71 diabetics grouped according to the presence or absence of PDI and data were obtained individually by a semi-structured questionnaire. PDI were classified using the UpToDate ${ }^{\circledR}$ database and the participants were compared according to socio-demographic and clinical variables through chi-square and $t$ student tests. Each participant used 4.6 drugs on average and over $70 \%$ of participants showed some PDI (total of 140 interactions). Over $70 \%$ of them required monitoring of pharmacotherapy and $6.4 \%$ of them would need to consider a change in therapy. PDI increased with the number of drugs prescribed $(\mathrm{p}<0.001)$ and did not change according to the gender, age and duration of DM2

Keywords: pharmacoepidemiology; drug interactions; diabetes mellitus; health education

\section{RESUMO}

Estudo transversal com objetivo de determinar a prevalência, impacto clínico e fatores associados a Interações Medicamentosas Potenciais (IMP) em portadores de diabetes mellitus tipo 2 (DM2) em participantes de um programa de educação em saúde. O estudo envolveu 71 diabéticos e os dados foram obtidos individualmente, por meio de um

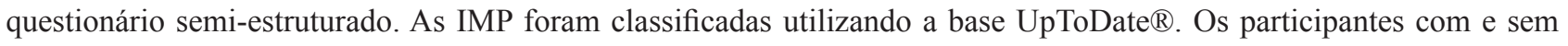
IMP foram comparados considerando variáveis sócio-demográficas e clínicas através dos testes qui-quadrado e t-student. Foram utilizados 4,6 medicamentos em média e mais de 70\% apresentaram alguma IMP (total de 140 interações). Mais de $70 \%$ requeriam monitoramento da farmacoterapia e em 6,4\% havia necessidade de considerar alteração da prescrição. As IMP aumentaram conforme número de medicamentos prescritos $(\mathrm{p}<0,001)$ e não variou quanto ao gênero, idade e tempo de diagnóstico.

Palavras chave: farmacoepidemiologia; interações medicamentosas; diabetes mellitus 


\section{INTRODUÇÃO}

Estudos epidemiológicos tem demonstrado importantes mudanças no perfil etiológico das doenças predominantes na população mundial. Essa transição epidemiológica é caracterizada por um aumento da incidência de doenças crônicas não transmissíveis (DCNT), dentre as quais se destaca a Diabetes Mellitus (DM) (1).

Em 2010, 285 milhões de pessoas entre 20 a 79 anos eram diabéticas, com uma prevalência mundial de $6,4 \%$; a estimativa é que, em 2030, esse número seja da ordem de 440 milhões, chegando à prevalência mundial de 7,7\% (2). Segundo o VIGITEL (Vigilância de Fatores de Risco e Proteção para Doenças Crônicas por Inquérito Telefônico), a frequência de adultos nas capitais brasileiras e no Distrito Federal que referiram o diagnóstico de DM em 2010 foi de $5,6 \%$ e $4,7 \%$, respectivamente (3).

A DM corresponde a uma doença onerosa devido a sua cronicidade, aos meios necessários para o seu controle e tratamento adequados e à gravidade de possíveis complicações associadas. A Federação Internacional de Diabetes (IDF) estimou que, em 2010, a DM seria responsável por $11,6 \%$ do total de gastos com saúde, chegando ao custo de 376 bilhões de dólares (4).

O objetivo do tratamento da DM é a manutenção dos valores glicêmicos em padrões o mais próximo possível da normalidade, evitando o surgimento de complicações micro e macrovasculares responsáveis pela morbidade da doença (5). Nesse contexto, o monitoramento do controle glicêmico através da medida de hemoglobina glicada (HbAlc) representa a principal ferramenta no monitoramento da DM em longo prazo (5).

A farmacoterapia envolvida no tratamento da DM pode envolver mais de um agente antidiabético (6). Além disso, a incidência de DM tipo 2 aumenta com o avanço da idade, assim como a probabilidade de ocorrência de comorbidades, tornando as intervenções clínicas mais complexas podendo exigir a prescrição de diversos tipos de medicamentos, caracterizando a polifarmácia. Esse processo, por sua vez, aumenta a probabilidade da ocorrência de algum tipo de interação medicamentosa potencial (IMP) $(6,7,8)$.

As IMP correspondem a eventos clínicos em que a ação de um medicamento sofre a interferên cia do efeito de outro medicamento $(7,9,10,11)$, resultando em redução ou aumento do efeito terapêutico ou na manifestação das reações adversas de um ou de outro medicamento (11).

Desse modo, as IMP podem resultar em fracasso terapêutico ou em efeitos adversos, podendo levar à hospita- lização do paciente ou mesmo ao aumento do tempo de permanência hospitalar, dependendo do nível de atenção em que o mesmo é atendido (11), bem como a não resolução ou agravamento da condição do paciente. Nesse contexto, esse trabalho teve como objetivo determinar a prevalência, o impacto clínico e os fatores associados às IMP em diabéticos tipo 2 participantes de um programa de educação em saúde.

\section{MÉTODO}

Foi realizado um estudo transversal com dados obtidos por meio de preenchimento de questionário pelos participantes do programa de educação em saúde Doce Desafio realizado no Centro Olímpico da Universidade de Brasília e nos postos de saúde $n^{\circ} 4$ de Samambaia - DF e $n^{\circ} 2$ de Sobradinho - DF durante o ano de 2009. Dos 90 participantes deste programa foram incluídos 71 na pesquisa que apresentavam os critérios de inclusão: ter diagnóstico de diabetes tipo 2 e estar em uso de 2 ou mais medicamentos.

O programa Doce Desafio inclui orientações relativas à prática de atividades físicas e acompanhamento dos demais aspectos que o tratamento da DM envolve, contando com a colaboração de profissionais e estudantes de educação física, farmácia, enfermagem, nutrição e medicina. As atividades são realizadas duas vezes por semana e tem duração de, aproximadamente, duas horas, durante as quais os participantes tem a pressão arterial e glicemia capilar aferidas antes e após a prática de exercícios físicos assistidos e recebem orientações coletivas sobre vários temas referentes à DM.

Os dados foram obtidos individualmente, por meio de um questionário semi-estruturado, aplicado por um grupo de estudantes de farmácia. Para levantamento do perfil clínico-epidemiológico, foram obtidos dados referentes à idade, tempo de DM, presença de comorbidades e medida de $\mathrm{HbA1C}$. A idade foi analisada de maneira contínua (média \pm DP) e em categorias (menor que 60 e maior ou igual a 60 anos), assim como o tempo de DM (categorias: de 1 a 4 , de 5 a 10 ou maior do que 10 anos). Em conformidade com a Sociedade Brasileira de Diabetes (SBD) (6), foram considerados com controle glicêmico adequado os participantes com $\mathrm{HbA} 1 \mathrm{C}$ menor ou igual a $7 \%$.

Os dados relativos aos medicamentos foram analisados através da classificação Anatomic Therapeutical Chemistry - ATC (12). Além disso, de acordo com o número de medicamentos em uso, a polifarmácia dos participantes foi classificada segundo Veehof e cols. (2000) em: leve (uso 
concomitante de 2 ou 3 medicamentos), moderada (uso concomitante de 4 a 5 medicamentos), ou grave (acima de 5 medicamentos) (13).

As IMP foram identificadas através da base de dados UpToDate ${ }^{\circledR}$ (14) e foram classificadas de acordo com a relevância clínica (risco) em três tipos: B (a IMP não requer intervenção clínica), C (IMP clinicamente significativa requerendo monitoramento da farmacoterapia) e D (IMP clinicamente significativa devendo-se considerar modificação da farmacoterapia). Quanto ao nível de evidência documentada, as IMP foram classificadas em tipos 1 (excelente: estudos controlados estabeleceram a existência da IMP), 2 (bom: a literatura sugere fortemente a existência da IMP, porém faltam estudos controlados), 3 (regular: poucas referências na literatura, porém considerações farmacológicas levam a suspeita da existência da IMP) e 4 (pobre: poucas referências na literatura, como relato de caso; poucos dados de suporte).

O teste t-student foi utilizado para comparar médias de dois grupos. Para verificação de associações entre variáveis categóricas foi utilizado o teste Qui-Quadrado. Os dados foram analisados através do software EpiInfo ${ }^{\circledR}$ versão 3.5.2. e os valores de p menores ou iguais a 0,05 foram considerados significantes.

A pesquisa foi aprovada pelo Comitê de Ética em Pesquisa da Faculdade de Ensino e Pesquisa em Ciências da Saúde do Distrito Federal sob número 020/09. As pessoas que concordaram em participar assinaram termo de consentimento livre e esclarecido.

\section{RESULTADOS}

A maioria dos participantes pertencia ao gênero feminino $(\mathrm{n}=51 ; 71,8 \%)$ e a média de idade amostral foi de $63,1 \pm 10,2$ anos (mínimo de 36 e máximo de 85 anos). O tempo médio de diagnóstico da DM foi de $8,9 \pm 6,8$ anos (mínimo de 1 e máximo de 31 anos). Quanto ao perfil clínico, 60 participantes $(84,5 \%)$ referiram ao menos uma comorbidade associada, com destaque à HAS $(80,3 \% ; n=57)$ e às dislipidemias $(35,2 \% ; n=25)$. A média de $\mathrm{HbA} 1 \mathrm{C}$ foi de $7,8 \pm 1,5 \%$, sendo que $60,6 \%$ dos participantes $(\mathrm{n}=43)$ apresentavam medida de $\mathrm{HbA1C}$ maior ou igual a $7 \%$.

A média de medicamentos em uso foi de 4,6 medicamentos (mínimo de 2 e máximo de 13) e os entrevistados relataram utilizar, no total geral, 93 diferentes medicamen- tos, sendo os mais referidos aqueles que atuam sobre o sistema cardiovascular $(n=41 ; 44,1 \%$ do total de medicamentos), seguidos pelos que atuam sobre o aparelho digestivo e metabolismo ( $\mathrm{n}=21 ; 22,6 \%$ do total de medicamentos). De acordo com Veehof e cols. (2000) (11), a polifarmácia foi classificada como leve em 26 (36,1\%) entrevistados, moderada em 25 (34,7\%) e grave em 20 (27,8\%).

A média de antidiabéticos orais (ADOs) em uso foi de $1,5( \pm 0,7$, com máximo de 4). Quanto ao regime terapêutico para tratamento da DM, 26 participantes $(36,0 \%)$ estavam em monoterapia oral, $28(38,9 \%))$ utilizavam mais de um ADO e $17(25,0 \%)$ estavam em insulinoterapia associada ou não a algum ADO, sendo 4 (5,5\%) deles em insulinoterapia plena.

Foram identificadas 140 IMP e $51(71,8 \%)$ participantes apresentaram pelo menos uma IMP, resultando em uma proporção de aproximadamente duas interações para cada diabético. Do total de IMP, 27 (19,3\%) foram classificadas como risco B, a maioria delas com evidência documentada regular (tipo 3) $(\mathrm{n}=22 ; 81,5 \%) ; 104$ (74,3\%) apresentavam risco C, 50 delas com boa documentação na literatura (tipo 2), e $9(6,4 \%$ ) eram risco D, com maioria regularmente evidenciada na literatura (tipo 3). Das 100 $(71,4 \%)$ IMP que apresentavam mecanismo de ação definido, a maior parte era farmacodinâmica $(n=75 ; 75,0 \%)$.

Das 27 IMP de risco B, 19 (70,4\%) envolvia um antidiabético oral (ADO) e um medicamento anti-hipertensivo pertencente à classe dos inibidores da enzima conversora de angiotensina (IECA).

Quanto às IMP risco $\mathrm{C}$, a maior parte um ADO e um anti-hipertensivo: $40,4 \%(n=42)$ com diurético tiazídico, 19 (45,2\%) delas com a hidroclorotiazida, e $11,5 \%(n=12)$ com beta-bloqueador, principalmente o propranolol.

Considerando as interações risco $\mathrm{D}$, a maioria era farmacocinética (6 das 7 com classificação conhecida na literatura) e possuía como consequências clínicas efeitos provenientes de um sinergismo de efeito adverso (5 das 9 IM risco D identificadas). As IMP risco B e C mais frequentes e todas as IMP risco $\mathrm{D}$, devido à sua menor proporção, estão descritas na Tabela 1.

Não houve diferença significativa na ocorrência de IMP entre os sexos $(p=0,338)$, assim como entre as diferentes faixas etárias consideradas $(\mathrm{p}=0,359)$. No que se refere à DM, os dois grupos de comparação não foram diferentes em relação ao tempo de diagnóstico de DM e nem em relação ao controle glicêmico (Tabela 2). 
Tabela 1. Aspectos relacionados às interações medicamentosas potenciais mais frequentes entre os participantes do Programa Doce Desafio

\begin{tabular}{|c|c|c|c|}
\hline Interação & Consequência & Documentação & Classificação \\
\hline \multicolumn{4}{|l|}{ Risco B $(n=27 ; 19,3 \%)$} \\
\hline Captopril + Gliclazida & $\begin{array}{l}\text { Aumento do efeito hipoglicemiante da } \\
\text { gliclazida }\end{array}$ & 3 & NE \\
\hline Enalapril + Gliclazida & $\begin{array}{l}\text { Aumento do efeito hipoglicemiante da } \\
\text { gliclazida }\end{array}$ & 3 & NE \\
\hline Amitriptilina + Indapamida & $\begin{array}{c}\text { Aumento do risco de prolongamento do } \\
\text { intervalo QT }\end{array}$ & 3 & Farmacodinâmica \\
\hline Enalapril + Glibenclamida & $\begin{array}{l}\text { Aumento do efeito hipoglicemiante da } \\
\text { glibenclamida }\end{array}$ & 3 & NE \\
\hline AAS + cálcio & Redução do efeito antiplaquetário do AAS & 1 & $\begin{array}{l}\text { Farmacocinética } \\
\text { (excreção) e } \\
\text { Farmacodinâmica }\end{array}$ \\
\hline \multicolumn{4}{|l|}{ Risco C ( $n=104 ; 74,3 \%)$} \\
\hline Indapamida + Metformina & $\begin{array}{l}\text { Redução do efeito anti-hiperglicemiante da } \\
\text { metformina }\end{array}$ & 2 & Farmacodinâmica \\
\hline Hidroclorotiazida + Metformina & $\begin{array}{l}\text { Redução do efeito anti-hiperglicemiante da } \\
\text { metformina }\end{array}$ & 2 & Farmacodinâmica \\
\hline Indapamida + Gliclazida & $\begin{array}{l}\text { Redução do efeito hipoglicemiante da } \\
\text { gliclazida }\end{array}$ & 2 & Farmacodinâmica \\
\hline AAS + Amitriptilina & Aumento do efeito antiplaquetário do AAS & 3 & Farmacodinâmica \\
\hline Amitriptilina + Gliclazida & $\begin{array}{l}\text { Aumento do efeito hipoglicemiante da } \\
\text { gliclazida }\end{array}$ & 3 & NE \\
\hline \multicolumn{4}{|l|}{ Risco D $(n=9 ; 6,4 \%)$} \\
\hline AAS + Naproxeno & $\begin{array}{l}\text { Aumento do risco de efeitos adversos/tóxicos } \\
\text { do AAS, como risco de sangramento }\end{array}$ & 2 & $\begin{array}{l}\text { Farmacocinética } \\
\text { (distribuição) }\end{array}$ \\
\hline Alendronato + Cálcio & Redução do efeito clínico do alendronato & 3 & $\begin{array}{l}\text { Farmacocinética } \\
\text { (absorção) }\end{array}$ \\
\hline Alopurinol + Captopril & $\begin{array}{c}\text { Aumento do risco de reações alérgicas ou de } \\
\text { hipersensibilidade ao alopurinol }\end{array}$ & 3 & NE \\
\hline $\begin{array}{l}\text { Amitriptilina + } \\
\text { Diidroergocristina }\end{array}$ & Aumento do risco de Síndrome Serotoninérgica & 3 & Farmacodinâmica \\
\hline Anlodipino + Sinvastatina & $\begin{array}{c}\text { Aumento do risco de efeitos adversos/tóxicos } \\
\text { da sinvastatina, como risco de miosite, } \\
\text { rabdomiólise }\end{array}$ & 3 & $\begin{array}{l}\text { Farmacocinética } \\
\text { (metabolismo) }\end{array}$ \\
\hline Cimetidina + Metformina & $\begin{array}{l}\text { Aumento do efeito anti-hiperglicemiante da } \\
\text { metformina }\end{array}$ & 1 & $\begin{array}{l}\text { Farmacocinética } \\
\text { (excreção) }\end{array}$ \\
\hline Enalapril + cálcio & Redução do efeito hipotensor do enalapril & 3 & NE \\
\hline Propiltiouracil + Varfarina & Redução do efeito anticoagulante da varfarina & 3 & $\begin{array}{l}\text { Farmacocinética } \\
\text { (metabolismo) }\end{array}$ \\
\hline Sinvastatina + Verapamil & $\begin{array}{c}\text { Aumento do risco de efeitos adversos/tóxicos } \\
\text { da sinvastatina, como risco de miosite, } \\
\text { rabdomiólise }\end{array}$ & 2 & $\begin{array}{l}\text { Farmacocinética } \\
\text { (metabolismo) }\end{array}$ \\
\hline
\end{tabular}

AAS: ácido acetil salicílico; NE: não especificada. 
DISCUSSÃO

O perfil epidemiológico da amostra foi semelhante ao encontrado em estudo realizado com diabéticos participantes de um programa de educação em saúde em São Paulo (15). Características sócio-demográficas exercem influência sobre a procura e utilização dos serviços de saúde e, nesse contexto, idosos e mulheres são os que mais demandam por estes serviços, sendo que as mulheres demonstram maior interesse em participar de ações preventivas em saúde $(16,17)$.

A frequência de polifarmácia na amostra foi elevada, com considerável proporção de diabéticos em polifarmácia grave. Entretanto, essa proporção foi menor do que a encontrada em estudo em São Paulo no qual mais de $85 \%$ dos diabéticos utilizavam mais de 5 medicamentos (18). Estes resultados são preocupantes, já que o uso de múltiplos medicamentos contribui para o aumento do custo e da complexidade do tratamento e aumenta o risco de incidência de Reações Adversas a Medicamentos (RAM) e de interações medicamentosas $(19,20)$. No presente estudo foi observado que a polifarmácia apresentou relação estatística significativa com a ocorrência de IMP, de modo que quanto maior o número de medicamentos em uso, maior a frequência da ocorrência de interações, resultado comum a outros estudos $(21,22)$.

A frequência de diabéticos que apresentaram pelo menos uma IMP foi inferior à encontrada em estudos realizados com idosos diabéticos e/ou hipertensos atendidos em nível de atenção primária, um em Santa Catarina (23) e outro em Goiás (24). No entanto, os resultados encontrados no estudo requerem atenção, já que mais de $70 \%$ dos diabéticos apresentaram ao menos uma IMP e mais de $80 \%$ delas eram clinicamente significativas (IMP risco C e risco D), sendo importante referir que no contexto em que essa pesquisa foi realizada, no qual é usual que os pacientes manifestem sinais e sintomas precoces o que leva muitas vezes a condutas e tratamentos empíricos (7).

Muitos problemas relacionados aos medicamentos são resultantes de IMP e, frequentemente, algumas manifestações clínicas são atribuídas a enfermidades quando, na verdade, estão relacionadas a essas potenciais interações (25). Quanto aos fatores possivelmente associados à ocorrência de interações, não houve uma diferença estatística significativa quando considerados os aspectos gênero, idade, tempo de DM e HbA1C. No entanto, como a literatura refere que esses aspectos, além daqueles relacionados aos medicamentos em uso, podem sim estar associados com a ocorrência de IMP (26), o quantitativo da amostra pode ter influenciado na ausência de resultados estatisticamente significativos.

Vale considerar, no entanto, que os diabéticos por conta da enfermidade e por conta das complicações possivel- mente associadas constituem um grupo com grande probabilidade de apresentarem interações medicamentosas $(27,28)$ e isso foi traduzido no presente estudo quando considerada a proporção de cerca de duas IMP para cada diabético.

Poucas IMP encontradas eram farmacocinéticas, corroborando assim com os dados encontrados na literatura que demonstram que interações farmacocinéticas são menos frequentes em pacientes que usam medicamentos para tratamento da DM, já que esses geralmente não são substratos, indutores ou inibidores de enzimas frequentemente envolvidas no metabolismo de medicamentos $(29,30)$. Em populações ditas gerais, esse tipo de interação é mais frequente, como evidenciado por Leão e cols. (2014), os quais referiram que $44,1 \%$ de um total de 279 IMP apresentadas por pacientes atendidos em nível de atenção primária em Vitória da Conquista-BA (7).

A maioria das IMP risco B apresentava como consequência direta a redução da glicemia, já que resultam em uma maior ação do ADO. A frequência desse tipo interação foi semelhante à encontrada por Amaral \& Perassolo (31) em estudo realizado no Rio Grande do Sul com diabéticos e hipertensos no contexto da APS. Porém, tais interações são pouco referenciadas na literatura científica e não requerem nenhuma ação clínica, sugerindo que seu impacto clínico não é significativo.

Quanto às IMP risco $\mathrm{C}$, que requerem um monitoramento da terapêutica, mais de $40 \%$ envolviam um ADO e um diurético tiazídico e tinham como consequência o aumento da glicemia resultante de uma redução do efeito do $\mathrm{ADO}$ envolvido. Não há dados na literatura que demonstrem a influência dos diuréticos tiazídicos sobre a efetividade da terapia antidiabética, porém, alguns trabalhos sugerem que esses medicamentos podem aumentar a resistência dos tecidos à insulina, resultando em menor efeito na redução da concentração de glicose sanguínea. Esse efeito parece estar associado à redução das concentrações de potássio induzida pelos diuréticos em questão $(32,33)$ e correspondeu a uma das várias hipóteses para justificar o mal controle glicêmico evidenciado em mais da metade do participantes foi justamente a potencial interação medicamentosa acima referida no estudo de Amaral \& Perassolo (2012) (31).

Ainda no contexto das IMP risco $\mathrm{C}$, vale ressaltar a considerável frequência de interações envolvendo beta -bloqueadores e ADO, especialmente as sulfonilureias. Estudos demonstram o benefício dos beta-bloqueadores em diabéticos, mas recomendam evitar os agentes não cardiosseletivos, como o propranolol, pois podem mascarar sinais fisiológicos importantes dos estados hipoglicêmicos, como a taquicardia $(29,34)$. Apesar da frequência de IMP risco $\mathrm{C}$ ter sido 11 vezes maior do que as de risco $\mathrm{D}$, cuja frequência foi semelhante à encontrada por Amaral \& Perassolo (2012) (31), vale considerar a importância dessa última, devido as suas potenciais consequências clínicas que estão associadas ao aumento do risco de reações adversas (11). 


\begin{tabular}{|c|c|c|c|c|c|}
\hline \multirow{3}{*}{ Variăveis } & \multicolumn{4}{|c|}{ IMP } & \multirow{3}{*}{ Valor de $\mathrm{p}^{*}$} \\
\hline & \multicolumn{2}{|c|}{$\operatorname{Sim}(n=20)$} & \multicolumn{2}{|c|}{ Não $(n=51)$} & \\
\hline & $\mathbf{N}$ & $\%$ & $\mathbf{N}$ & $\%$ & \\
\hline \multicolumn{6}{|l|}{ Gênero } \\
\hline Feminino & 16 & 75,0 & 35 & 68,6 & \\
\hline Masculino & 4 & 25,0 & 16 & 31,4 & 0,338 \\
\hline Idade (Média \pm DP) & \multicolumn{2}{|c|}{$61,9 \pm 9,6$} & \multicolumn{2}{|c|}{$63,6 \pm 10,4$} & 0,286 \\
\hline$<60$ anos & 9 & 45,0 & 17 & 33,3 & 0,359 \\
\hline$\geq 60$ anos & 11 & 55,0 & 34 & 67,7 & 0,295 \\
\hline Tempo de DM (Média \pm DP) & \multicolumn{2}{|c|}{$8,5 \pm 7,1$} & \multicolumn{2}{|c|}{$9,0 \pm 6,7$} & \\
\hline$<5$ anos & 5 & 25,0 & 16 & 32,0 & 0,207 \\
\hline 5-9 anos & 10 & 50,0 & 14 & 28,0 & \\
\hline$\geq 10$ anos & 5 & 25,0 & 20 & 40,0 & \\
\hline HbA1C (Média \pm DP) & \multicolumn{2}{|c|}{$8,0 \pm 1,6$} & \multicolumn{2}{|c|}{$7,7 \pm 1,5$} & 0,340 \\
\hline$<7 \%$ & 7 & 35,0 & 21 & 41,2 & \\
\hline$\geq 7 \%$ & 13 & 65,0 & 30 & 58,8 & 0,632 \\
\hline \multicolumn{6}{|l|}{ Polifarmácia } \\
\hline Leve & 17 & 85,0 & 9 & 17,7 & \\
\hline Moderada & 3 & 15,0 & 22 & 43,1 & \\
\hline Grave & 0 & 0,0 & 20 & 39,2 & $<0,001$ \\
\hline
\end{tabular}

HbA1C: Hemoglobina glicada; IMP: Interação Medicamentosa Potencial.

* Teste $\chi 2$ para variáveis categóricas e teste t student para variáveis contínuas.

Há ainda muitas questões a serem respondidas sobre a ação de fatores protetores ou que predispõem pacientes a interações medicamentosas, o que torna a detecção de uma IMP e de suas consequências clínicas na prática mais difícil. Porém, algumas medidas podem ser tomadas para reduzir o risco de IMP, como a escolha de medicamentos que não estejam envolvidos em interações clinicamente relevantes ou mesmo a realização de ajuste de dose, caso a associação que leve à interação seja necessária (11).

A prática da polifarmácia é frequente em diabéticos devido às características da doença e à elevada frequência de comorbidades. Este fato contribui para a ocorrência de interações medicamentosas que aumentam a probabilidade da ocorrência das RAM, levando ao fenômeno conhecido como cascata de prescrição, comum em idosos, os quais são mais sensíveis aos efeitos terapêuticos e tóxicos dos medicamentos $(19,20)$.

É imprescindível, portanto, o monitoramento da farmacoterapia, valorizando a avaliação de IMP, de efeitos colaterais, queixas inespecíficas e efeitos adversos do tratamento. $\mathrm{O}$ seguimento farmacoterapêutico realizado por farmacêutico inserido na equipe de saúde pode contribuir muito para identificar potenciais riscos de ocorrência de RAM e auxiliar no ajuste terapêutico, desempenhando papel fundamental para a promoção do uso racional de medicamentos e o controle da doença, aumentando a qualidade de vida dos pacientes (35).

A literatura mostra que as manifestações clínicas das IMP são pouco ou mesmo não específicas, podendo mimetizar sinais e sintomas de enfermidades (36). Nesse contexto, são essenciais ações de conscientização e constante qualificação de todos os profissionais envolvidos nos processos que vão desde a avaliação e prescrição à administração dos medicamentos, passando pelo ato de dispensação dos mesmos com a devida orientação ao usuário, estabelecendo, dessa forma a atenção farmacêutica.

As principais limitações do presente estudo estão relacionadas ao seu tipo, já que corresponde a um estudo transversal passível de viés seleção e de informação, considerando a influência do estado emocional do diabético no momento da entrevista e/ou falta de conhecimento de sua condição clínica. Outra limitação se refere ao fato de não 
se conseguir aferir a real importância clínica das interações detectadas. Ressalta-se, ainda, que a frequência de IMP encontrada pode estar subestimada, uma vez que não foram incluídos neste estudo os medicamentos isentos de prescrição, plantas medicinais ou remédios caseiros.

\section{CONCLUSÃO}

O seguimento farmacoterapêutico realizado por farmacêutico inserido na equipe de saúde pode contribuir muito para identificar potenciais riscos de ocorrência de RAM associadas a possíveis interações medicamentosas, como as evidenciadas no presente estudo. Isso possibilitaria ajustes terapêuticos, e, dessa forma, a promoção do uso racional de medicamentos e o controle da enfermidade, aumentando a qualidade de vida dos pacientes, aspectos importantes quando se fala em DCNT.
São necessários mais estudos que avaliem a utilização de medicamentos que forneçam dados sobre as prescrições com foco nos desfechos clínicos de interações medicamentosas, que podem contribuir para o estabelecimento de estratégias de ação para prevenção, visando reduzir a proporção e/ou possíveis impactos clínicos de IMP.

\section{AGRADECIMENTOS:}

Agradecimentos ao apoio dos diabéticos participantes do estudo. Agradecimentos também pela bolsa disponibilizada pelo Conselho Nacional de Desenvolvimento Científico e Tecnológico (CNPq) e pelo apoio para manutenção do programa pela Fundação de Apoio à Pesquisa do Distrito Federal (FAP-DF).

MC. Avaliação das Interações Medicamentosas em Prescrições Hospitalares de Pacientes Sob Uso de Anti-Hipertensivos. Lat Am J Pharm. 2009. 28 (4): 501-6.

11. BRASIL. Ministério da Saúde. Formulário Terapêutico Nacional. Brasília (DF): Editora do Ministério da Saúde; 2008.

12. OMS. Organização Mundial da Saúde. WHO Collaborating Centre for Drug Statistics Methodology. 2012. [Citado 2014 Maio 10]. Disponível em: http://www.whocc.no/ atc ddd index/.

13. Veehof ${ }^{-}$LJG, Stewart RE, Haaijer-Ruskamp FM, Meyboonde Jong B. The development of polypharmacy. A longitudinal study. Fam Pract. 2000. 17: 261-7.

14. UpToDate: a clinical decision support system that helps clinicians throughout the world provide the best patient care. 2012. [Citado 2014 Fev 20]. Disponível em: http:// www.uptodate.com/contents/search.

15. Zanetti ML, Obtero LM, Biaggi MV, Santos MA, Péres DS, Guimarães FPM. Satisfação do paciente diabético em seguimento em um programa de educação em diabetes. Rev Lat-Am Enferm. 2007.15 (4): 583-9.

16. Ribeiro RA, Mello RGB, Melchior R, Dill JC, Hohmann $\mathrm{CB}$, Lucchese AM, et al. Custo anual do manejo da cardiopatia isquêmica crônica no Brasil. Arq Bras Cardiol. 2005. 85 (1): 3-8.

17. Pappa E, Niakas D. Assessment of health care needs and utilization in a mixed public-private system: the case of the Athens area. BMC Health Serv Res. 2006. 6 (146): $1-11$.

18. Nascimento AB, Chaves EC, Grossi SAA, Lottenberg SA. A relação entre polifarmácia, complicações crônicas e depressão em portadores de Diabetes Mellitus Tipo 2. Rev Esc Enferm USP. 2010. 44 (1): 40-6.

19. Austin RP. Polypharmacy as a Risk Factor in the Treat- 
ment of Type 2 Diabetes. Dia Spectr. 2006. 19 (1): 13-6.

20. Ribeiro AQ, Acurcio FA, Wick JY. Pharmacoepidemiology of the elderly in Brazil: state of the art. Consult Pharm. 2009. 24: 30-6.

21. Mibielli P, Rozenfeld S, Matos GC, Acurcio FA. Interações medicamentosas potenciais entre idosos em uso dos anti-hipertensivos da Relação Nacional de Medicamentos Essenciais do Ministério da Saúde do Brasil. Cad. Saúde Pública. 2014. 30 (9): 1947-56.

22. Secoli SR, Figueras A, Lebrão ML, Lima FD, Santos JLF. Risk of potential drug-drug interactions among Brazilian elderly. Drugs Aging. 2010. 27: 759-70.

23. Codagnone Neto V, Garcia VP, Santa Helena ET. Possible pharmacological interactions in hypertensive and/or diabetic elderly in family health units at Blumenau (SC). Braz J Pharm Sci. 2010.46 (4): 795-804.

24. Tavares MS, Macedo TC, Mendes DRG. Possíveis Interações Medicamentosas em um Grupo de Hipertenso e Diabético da Estratégia Saúde da Família. Revista de Divulgação Científica Sena Aires. 2012. 2: 119-126

25. Sehn R, Camargo AL, Heineck I, Ferreira MBC. Interacções medicamentosas potenciais em prescrições de pacientes hospitalizados. Infarma. 2003. 15(9-10): 77-81.

26. Monteiro C, Marques FB, Ribeiro CF. Interacções Medicamentosas como causa de iatrogenia evitável. Rev Port Clin Geral. 2007. 23: 63-73.

27. Marquito AB, Fernandes NMS, Colugnati FAB, Paula $\mathrm{RB}$. Interações medicamentosas potenciais em pacientes com doença renal crônica. J. Bras. Nefrol. 2014. 36 (1): 26-34.

28. Freeman JS, Gross B. Potential drug interactions associa- ted with treatments for type 2 diabetes and its comorbidities: a clinical pharmacology review. Expert Rev Clin Pharmacol. 2012. 5 (1): 31-42.

29. Kroner BA. Common Drug Pathways and Interactions. Dia Spectr. 2002. 15 (4): 249-55.

30. Scheen AJ. Drug interactions of clinical importance with antihyperglycaemic agents: an update. Drug Saf. 2005. 28(7): 601-31.

31. Amaral DMD, Perassolo MS. Possíveis interações medicamentosas entre os anti-hipertensivos e antidiabéticos em participantes do Grupo HIPERDIA de Parobé, RS (Uma análise teórica). Rev Ciênc Farm Básica Apl. 2012. 33(1): 99-105.

32. Salvetti A, Ghiadoni L. Thiazide Diuretics in the Treatment of Hypertension: An Update. J Am Soc Nephrol. 2006. 17: 25-9.

33. Israili ZH, Lyoussi B, Hernandez-Hernandez R, Velaco M. Metabolic Syndrome: Treatment of Hypertensive Patients. Am J Ther. 2007. 14: 386-402.

34. Huynh K, Bernardo BC, McMullen JR, Ritchie RH. Diabetic cardiomyopathy: Mechanisms and new treatment strategies targeting antioxidant signaling pathways. Pharmacology \& Therapeutics. 2014. 142: 375-415.

35. Miller L, Pater KS, Corman S. The role of clinical decision support in pharmacist response to drug-interaction alerts. Research in Social and Administrative Pharmacy. 2015. 11 (3): 480-6

36. Varallo FR, Costa MA, Mastroianni PC. Potenciais interações medicamentosas responsáveis por internações hospitalares. Rev Ciênc Farm Básica Apl., 2013. 34(1): 79-85 